\title{
Ultrasonically Assisted Anchoring of Biodegradable Implants for Chevron Osteotomies - Clinical Evaluation of a Novel Fixation Method
}

\author{
Kai Olms ${ }^{1}$, Thorsten Randt ${ }^{1}$, Nils Reimers ${ }^{2}$, Nils Zander ${ }^{3}$ and Arndt P. Schulz ${ }^{*}, 4$ \\ ${ }^{1}$ Praxisklinik Am Park, Bad Schwartau, Germany \\ ${ }^{2}$ University of Applied Sciences, Lübeck, Germany \\ ${ }^{3}$ Stryker Osteosynthesis Kiel, Germany \\ ${ }^{4}$ Department of Orthopedics and Trauma, University Hospital Lübeck, UKSH, Ratzeburger Allee 160, D-23538 Lübeck, \\ Germany
}

\begin{abstract}
Reconstructive osteotomies for the treatment of Hallux valgus are among the most prevalent procedures in foot and ankle surgery.

The combination of biodegradable materials with an innovative method for fixation by application of ultrasonic energy facilitates a new bonding method for fractures or osteotomies. As clinical experience is still limited, the aim of this study was to assess the safety and performance of the SonicPin system for fixation of Austin/Chevron osteotomies.

Chevron osteotomy was performed on 30 patients for the treatment of Hallux valgus. The used SonicPins were made from polylactide and are selectively melted into the cancellous bone structure during insertion by ultrasonic energy. Patients were followed for one year, which included X-ray and MRI examinations as well as evaluation of life quality by EQ-5D (EuroQol).

The MRI after three months showed adequate bone healing in all cases and no signs of foreign body reactions, which was again confirmed by MRI 12 months postoperatively. The bony healing after 12 months was uneventful without any signs of foreign body reactions.

In summary, based on the low complication rate and the significant improvement in health related quality of life (EQ-5D) reported in this study, fixation of an Austin/Chevron osteotomy with a SonicPin for treatment of Hallux valgus can be considered to be safe and efficient over the short term.
\end{abstract}

Level of Clinical Evidence: Therapeutic Level III.

Keywords: Biodegradable implants, chevron osteotomy, Hallux valgus, Polylactide pins, ultrasound activation.

\section{INTRODUCTION}

In 1981, the Chevron osteotomy was introduced by Austin and colleagues [1] and has become widely accepted for correcting mild to moderate Hallux valgus deformities [2]. Although originally internal fixation was considered not to be necessary with this procedure, fixation as an aid for stability is nowadays generally favored. Fixation is usually performed using small fragment screws, K-wires and other metallic implants $[3,4]$. Resorbable fixation methods have been used in the past as an attempt to reduce revisions for screw removal but have never been broadly accepted [5-8]. In contrast to the initial in vitro results [9], the clinical experience with theses implants could not confirm the intended degradation characteristics of the materials. Implant loosening with subsequent soft tissue irritations and foreign

*Address correspondence to this author at the Department of Orthopedics and Trauma, University Hospital Lübeck, UKSH, Ratzeburger Allee 160, D-23538 Lübeck, Germany; Tel: +494515002642;

E-mail: schulz@biomechatronics.de body reactions were caused by non-physiological absorption processes [10].

Although generally good clinical results are achieved with the Chevron osteotomy procedure [11, 12], complications are still reported in up to $20 \%[13,14]$. The revision rate has been reported between 2.5 to $5.6 \%$ [15-17], mainly for removal of metal work due to metal irritation or patient request $[15,18]$. In a recent study, removal of metal work was necessary in $9.2 \%$ of cases after first ray surgery, resulting in a significantly better Visual Analogue ScaleFoot and Ankle questionnaire result after the revision [18]. The necessity of a further surgical procedure thereby has a significant socio-economic and social impact.

Since a few years, a method for osteosynthesis of small bones has been described that combines poly-lactide screwpins with an ultrasound assisted technique that relies on short time liquidification and intraosseous anchoring $[19,20]$. This technique, initially termed "bone welding", uses polylactides optimized for the use in small bone surgery, especially with regards to their degradation properties [21-23]. 
Biomechanical and in-vivo studies could prove the suitability for small bone surgery [19, 21, 24, 25]. After successful use in craniofacial surgery [26-28], this implant has recently been introduced to the orthopedic market under the name "SonicPin" (Stryker Osteosynthesis).

The idea behind our study was to evaluate whether fixation of the Chevron procedure is possible in a safe and reliable manner with this device, possibly resulting in a higher patient satisfaction and a lower revision rate.

The objective of this clinical study was thereby to prove the general safety and the capability of the SonicPin to maintain alignment and fixation of an Austin/Chevron osteotomy without occurrence of foreign body reactions. The clinical outcome of the procedure as experienced by the patients over the medium term will be evaluated by a health related quality of life assessment using the well-described EQ-5D questionnaire $[16,29,30]$. Comparison to other methods and long-term results are not part of this study.

\section{PATIENTS AND METHODS}

This study was designed as a prospective consecutive open-arm cohort evaluation to assess the safety and performance of the SonicPin. Study center was a dedicated clinic for foot surgery.

The clinical investigation protocol was reviewed and approved by the local ethics committee (Number: III/EK53/08). All subjects included in this study provided written Informed Consent and met all inclusion criteria.

The study has been conducted in accordance with ISO 14155 "Clinical investigation of medical devices for human subjects". The protection of the rights of patients participating in the study was in accordance with the Declaration of Helsinki. The study comprised of 30 consecutive patients enrolled from May $5^{\text {th }}$ to September $11^{\text {th }}$ 2008 with a mild to moderate Hallux valgus deformity.

Patients between 18 and 80 years of age with a diagnosed mild to moderate hallux valgus deformity with an intermetatarsal angle below $16^{\circ}$ with or without concomitant forefoot deformities and the indication for a Chevron procedure were included. Furthermore, the mental ability to give informed consent and the ability to follow the postoperative instructions was required.

Patients pre-operatively classified as ASA IV, patients with active foot joint or systemic infection, patients with a systemic illness that might interfere with the well-being of the patient and affect the outcome of the procedure (like Paget-syndrome, renal osteodystropy, systemic-immune disorder, vaculitis), Patients with metabolic bone disease, e.g., severe osteoporosis, Ricket's disease, patients with gout, rheumatoid arthritis, or neoplastic diseas and patients with a known allergy to Poly-L-lactide and Poly D, 30\% Llactide (PLDLA) were excluded. Patients who were known to be pregnant at the time of enrollment or planned to become pregnant in the coming 12 months were also excluded. Patient demographics are given in (Table 1).

The ultrasound-assisted anchoring technology is a novel method for anchoring thermoplastic biodegradable pins in bone. The technique applies ultrasonic energy to the polymer, inducing heat-generating shearing forces at the contact interface between implant and bone. During this, the polymer is liquefied and penetrates the cavities of the surrounding bone. Within seconds, the polymer solidifies again and forms a strong and uniform anchorage between implant and bone [31]. For this study the ultrasound applicator "Sonic fusion" was used (Stryker Osteosynthesis, Schönkirchen, Germany) for the implantation of biodegradable pins (2.0 mm diameter, length 22-26 mm), made of poly(L-lactide-co-D,L-lactide) 70:30 (SonicPin, Stryker Osteosynthesis, Schönkirchen, Germany) (Fig. 1A, B).

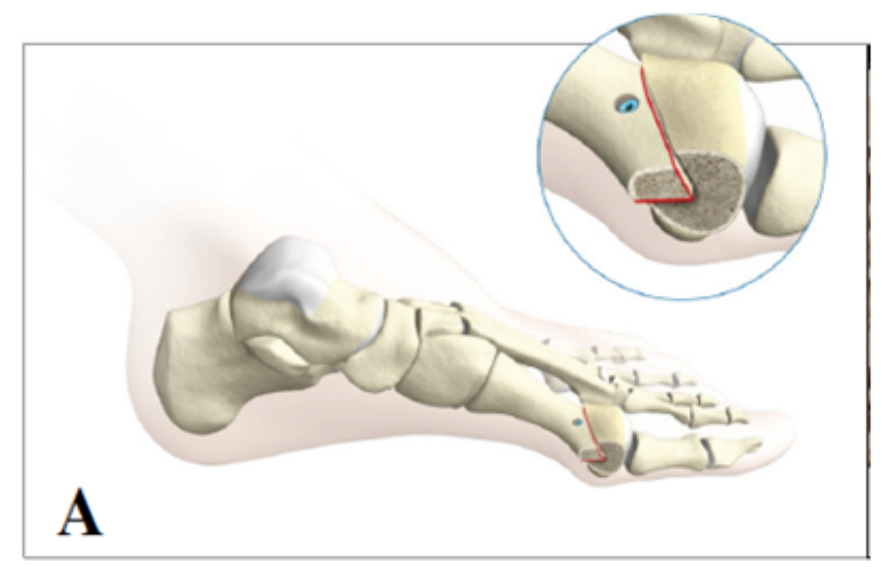

Fig. (1A). Austin/chevron osteotomy with lateral shift and fixation (Stryker Product Information SonicPin, Literature Number: 90-17100).

All procedures were performed by a single surgeon. The procedure was carried out standardized in the following way: A dorsal medial skin incision was made medial to the extensor hallucis longus crossing the first metatarsophalangeal joint. If indicated, a lateral release was performed through the same incision, including the release of the adductor tendon and the fibular sesamoidal ligament (12 patients). A T-shaped incision of the capsule was performed, thus exposing the joint. Following the resection of the medial

Table 1. Patient demographics.

\begin{tabular}{|c|c|c|c|c|}
\hline & Age [Years] & Height $[\mathbf{c m}]$ & Weight $[\mathbf{k g}]$ & BMI [kg/m $\mathbf{m}^{2}$ \\
\hline \hline Mean & 53.3 & 167 & 71.5 & 25.7 \\
\hline Median & 52.5 & 167 & 73.0 & 25.6 \\
\hline Maximum & 79.0 & 182 & 96.0 & 32.9 \\
\hline Minimum & 19.0 & 154 & 52.0 & 19.6 \\
\hline Standard Deviation & 14.62 & 8 & 12.2 & 3.8 \\
\hline
\end{tabular}


eminence, a K-wire was inserted in the centre of the metatarsal head. A V-shaped osteotomy was performed at an angle of 60 degrees with the apex at the K-wire followed by the lateral translation of the capital fragment.

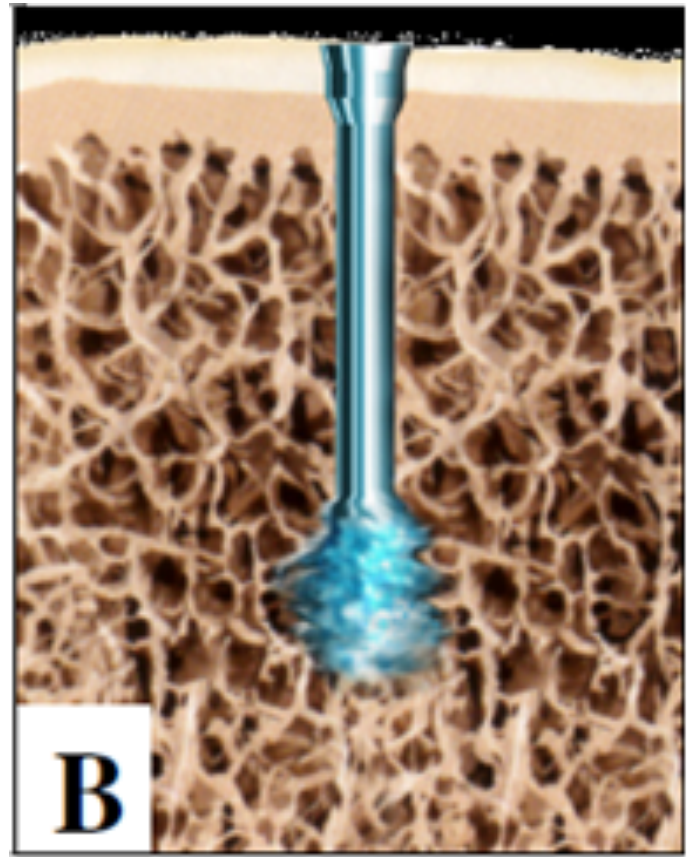

Fig. (1B). Fixation principle of the SonicPin: During insertion into a predrilled hole, the tip of the pin is melted by ultrasound, resulting in interdigitation of the SonicPin with the surrounding cancellous bone (Stryker Product Information SonicPin, Literature Number: 90-17100).

Prior to the preparation of the pilot hole, a $0.8 \mathrm{~mm}$ $\mathrm{K}$-wire was inserted across the osteotomy to determine the position, direction and length of the pin. As the K-wire was penetrating the cartilage of the metatarsal head, the position and direction of the definitive implant could be verified visually. The K-wire was then retracted until it was not visible any more and the length could now be determined using the dedicated depth gauge. After choosing the appropriate drill length, the K-wire was removed and its entrance point was used for the drill hole for the SonicPin. The SonicPin of the appropriate length was mounted on the hand piece and placed into the drill hole crossing the osteotomy. Ultrasound was activated for two to three seconds and the SonicPin was introduced simultaneously applying moderate axial force until complete insertion. The completion of the process was indicated by an acoustic signal after five seconds and the hand piece was disconnected from the SonicPin. If required, hammer toe, bunionette or hallux rigidus was treated accordingly if required during the same procedure (Table 2). After resection of redundant bone, the incision was closed. The postoperative protocol allowed for immediate full weight bearing while the use of crutches was recommended for patient's comfort.

Patients were evaluated before surgery, at the day of surgery, at day 1 after the procedure, 6 weeks, 3 months, and 12 months after surgery, assessing safety and performance of the implant by clinical examination, evaluation of complications, EQ-5D questionnaire (preoperatively, 6 weeks, 3 months, 12 months) [32, 33], X-ray examinations (medial/lateral and anterior/posterior views; preoperatively, day 1, 6 weeks), and MRI-evaluations (3 and 12 months).

Table 2. Procedure time.

\begin{tabular}{|c|c|c|}
\hline Treatment & $\mathbf{n}$ & OP/Time [Median] \\
\hline \hline Hallux valgus & 19 & 31 \\
\hline Hallux valgus with hammer toe & 9 & 39 \\
\hline Hallux valgus with bunionette & 1 & 33 \\
\hline Hallux valgus et. rigidus & 1 & 34 \\
\hline
\end{tabular}

Health related quality of life assessment was determined using the EQ-5D questionnaire at a preoperative baseline, 6 weeks, 12 weeks, and 12 months after the index procedure.

One patient could not be followed up completely due to a non-device related lethal myocardial infarction 7 months postoperatively; complete datasets are therefore available for 29 patients. Outcome assessment was independent of the surgeon who performed the procedure, all data was monitored.

Evaluation of the items was performed independent of the surgical team. Data was sampled in a spreadsheet and transferred to SPSS (Version 17, IBM-SPSS, Chicago, USA) for statistical evaluation.

\section{RESULTS}

In all cases, the fixation with a sonic pin was possible. Due to incomplete melting of the pin an additional pin was applied for safety reasons twice $(6.7 \%)$.

The median operation time (skin-to-skin) was 33.5 minutes (range: 18 to 57 minutes, Table 2).

There were 3 irregularities in the radiological follow-up. At three months follow-up, one patient (3.3\%) showed fragment dislocation of $2 \mathrm{~mm}$ on conventional radiographs, possibly due to instability of the pin. The MRI after three months showed no signs of foreign body reactions. At 12 months follow-up, the same patient showed fracture of the pin with complete bone healing without clinical problems.

One case $(3.3 \%)$ showed an unusual bone healing pattern without clinical correlation. The MRI revealed signs of edema around the regularly shaped implanted pin and cortical bone defect dorsally at three months follow-up (Fig, $\mathbf{2 A}, \mathbf{B})$. A radiograph was taken six months postoperatively, which showed complete bony healing with intraosseous sclerosis (Fig. 3).

In another case with a moderate Hallux rigidus component $(3.3 \%)$, the patient complained of arthritic plantar pain in the first MPJ at the 12 month follow-up examination. The MRI showed an increase of the arthritis, which will probably require an arthrodesis in the future (Fig. 4A, B).

At 12 months postoperatively, 29 MRI examinations were collected with no changes to the findings after three months. The implant could be detected without signs of resorption in all 29 subjects. One representative case with all 
radiographic examinations (i.e., preoperative, after one day, six weeks: X-rays; three and 12 months: MRI) is shown in (Figs. 5-9).

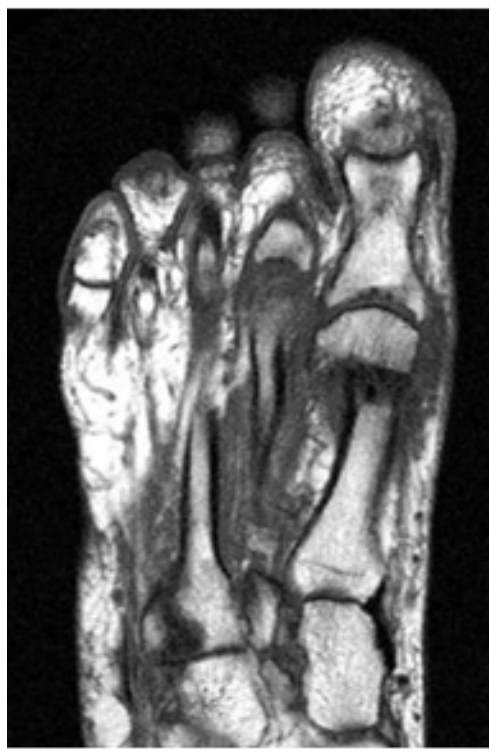

Fig. (2A). Postoperative anteroposterior MRI of a female patient, three months after surgery with a possible sign of edema.

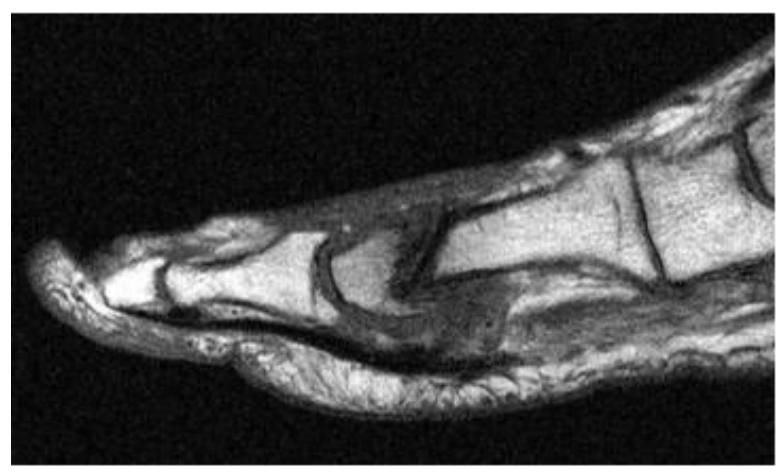

Fig. (2B). Postoperative lateral MRI of a female patient, three months after surgery.

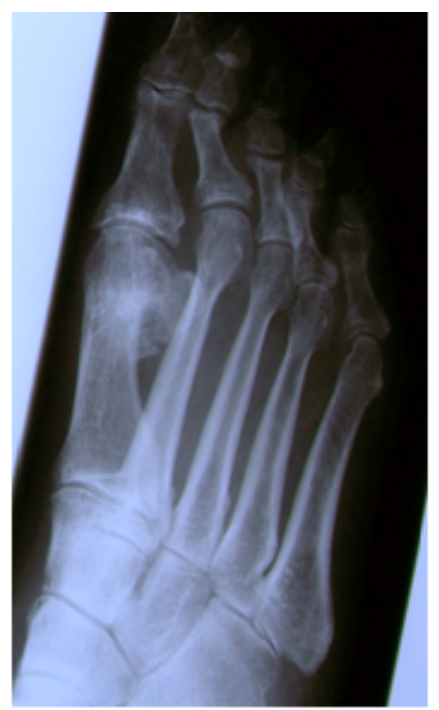

Fig. (3). Postoperative anteroposterior radiograph of a female patient, six months after surgery.

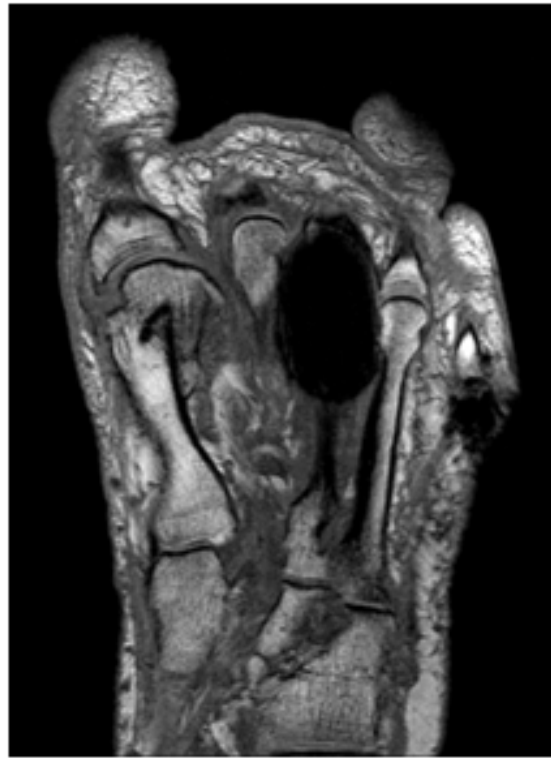

Fig. (4A). Postoperative anteroposterior MRI of a female patient, three months after surgery.

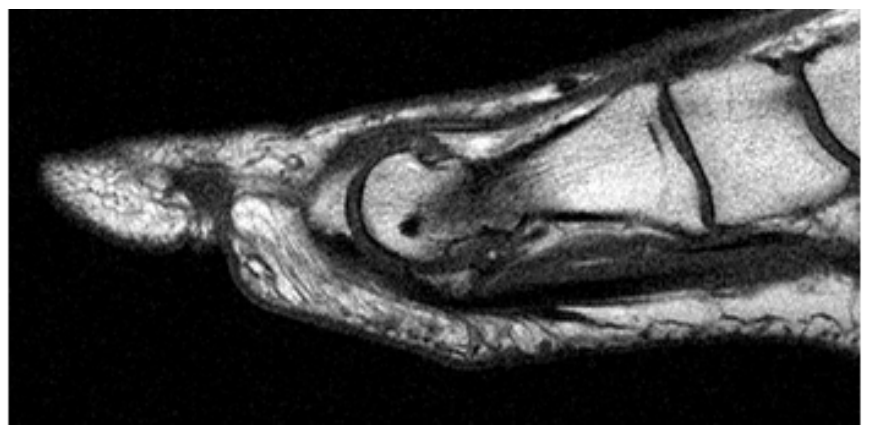

Fig. (4B). Postoperative lateral MRI of a female patient, three months after surgery.

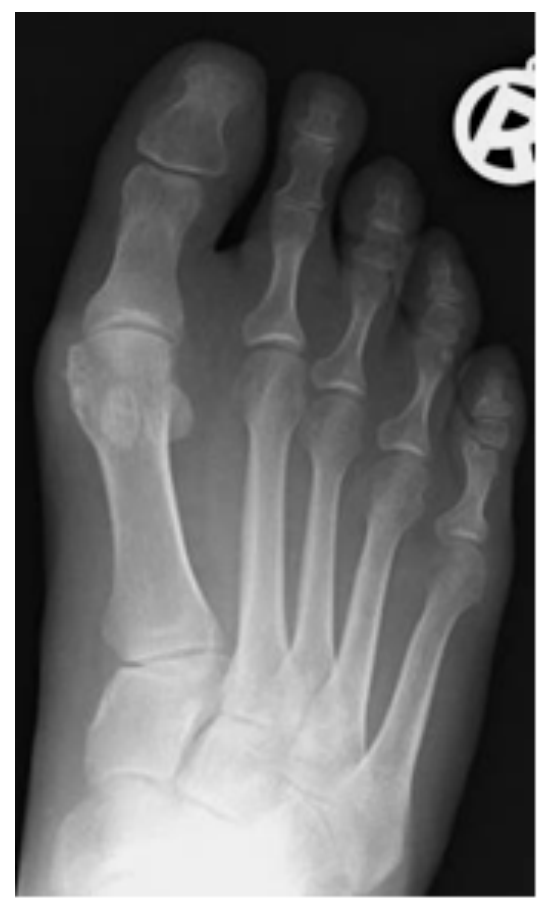

Fig. (5A). Preoperative anteroposterior radiograph of a female patient. 


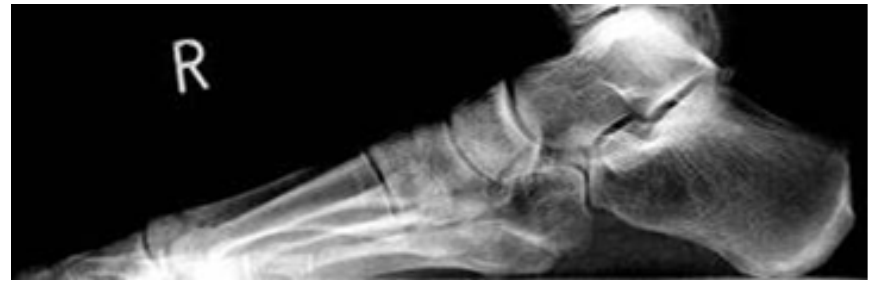

Fig. (5B). Preoperative lateral radiograph of a female patient.

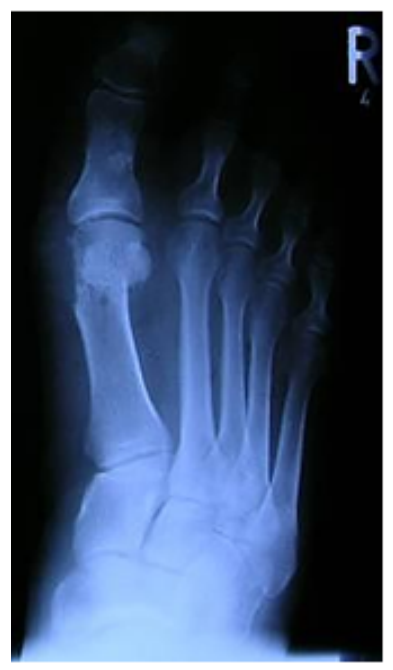

Fig. (6A). Postoperative anteroposterior radiograph of a female patient, 1 day after surgery.

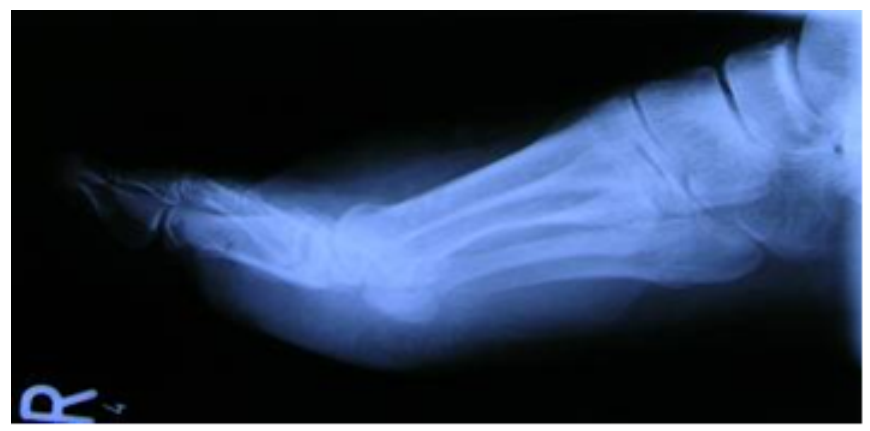

Fig. (6B). Postoperative lateral radiograph of a female patient, one day after surgery.

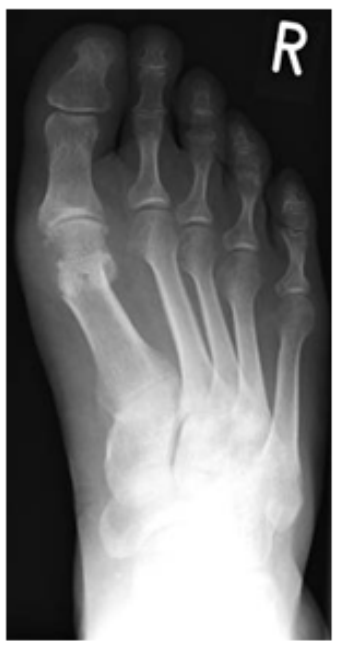

Fig. (7A). Postoperative anteroposterior radiograph of a female patient, six weeks after surgery.

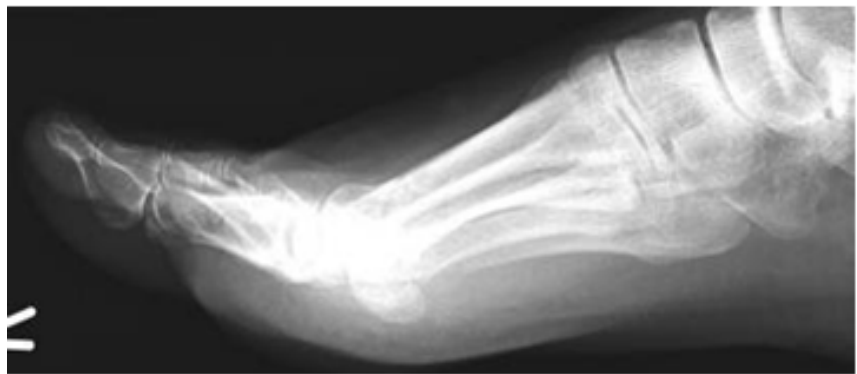

Fig. (7B). Postoperative lateral radiograph of a female patient, six weeks after surgery.

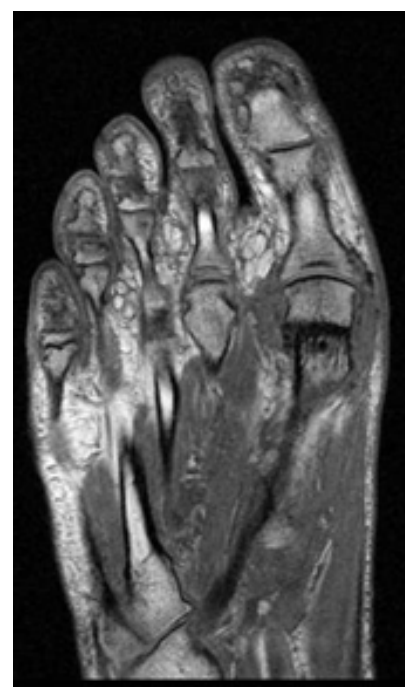

Fig. (8A). Postoperative anteroposterior MRI of a female patient, three months after surgery.

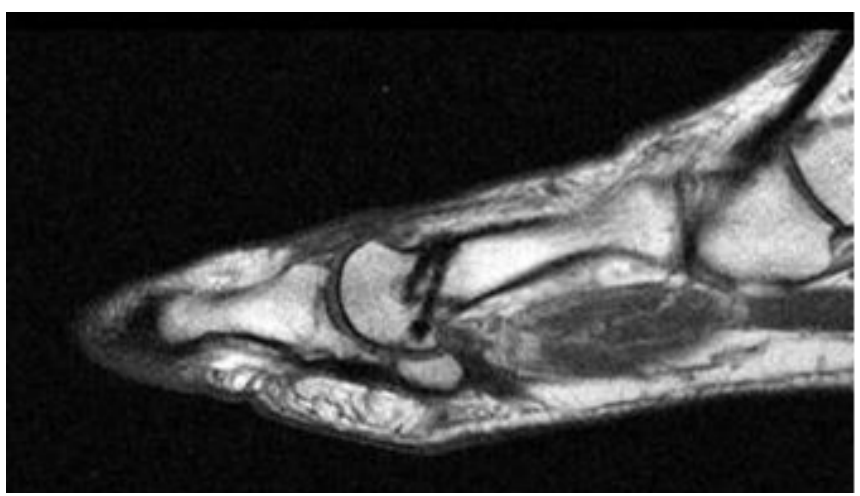

Fig. (8B). Postoperative lateral MRI of a female patient, three months after surgery.

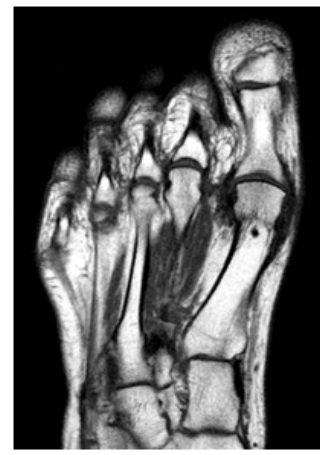

Fig. (9A). Postoperative anteroposterior MRI of a female patient, 12 months after surgery. 


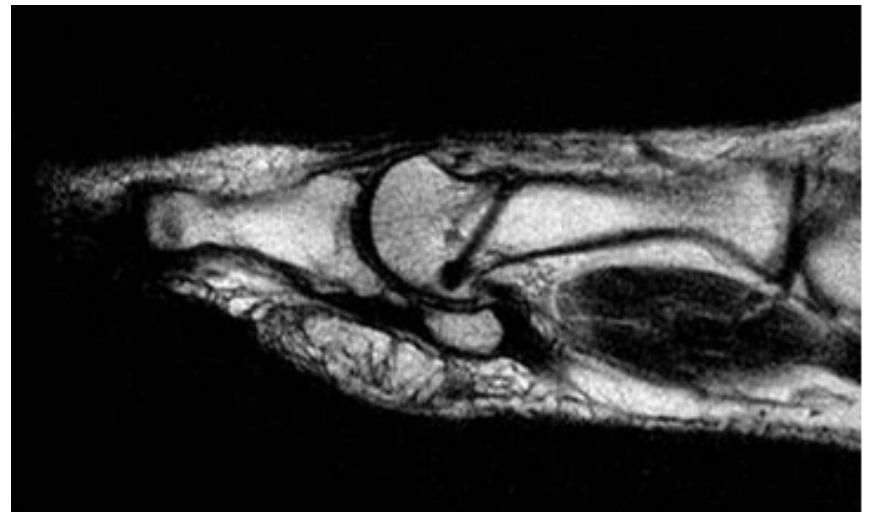

Fig. (9B). Postoperative lateral MRI of a female patient, 12 months after surgery.

Screening examination and questionnaires revealed a median baseline EQ-5D of 0.727 (range: 0.193 to 1.0 ) and a median rated health of $85 \%$ (range: 40 to $100 \%$ ). At six weeks postoperatively, the median EQ-5D was 0.796 (range: 0.727 to 1 ), and the median rated health was $90 \%$ (range: 70 to $100 \%$ ). At three months postoperatively, the median EQ5D was 1.0 (range: 0.727 to 1 ), and the median rated health was $95 \%$ (range: 75 to $100 \%$ ). At 12 months postoperatively, the median EQ-5D was 1.0 (range: 0.727 to 1 ), and the median rated health was $100 \%$ (range: 80 to $100 \%$ ). EQ-5D and rated health values are shown in Fig. (10). The improvements compared to the baseline values (screening) are significant (six weeks: $\mathrm{p}=0.001$, three months: $\mathrm{p}<$ 0.001, 12 months: $p<0.001)$, Wilcoxon Signed Ranks Test).

\section{DISCUSSION}

We could show in our study that the use of the SonicPin method for fixation of a Chevron procedure is safe and gives reliable results in short term evaluation. Over the course of the study, the revision rate was zero, a good quality of life, as assessed by the EQ-5D, was achieved.

Given the high incidence of Hallux valgus, Austin/Chevron osteotomy is a frequently performed procedure in foot and ankle surgery [34]. Fixation is used routinely and mainly achieved utilizing small metallic screws [2, 4, 12, 25, 35-37]. As stated in the introduction, removal of metal works is not uncommon after this operation $[36,37]$. Polylactides as biodegradable materials have been used as screws and press-fit pins in the past for this procedure but have been largely abandoned due to foreign body reactions and soft tissue irritations subsequent to implant loosening [37]. To address the soft tissue reactions, the investigational device of this study utilizes a second generation of polylactide with adapted resorption characteristics [23], to avoid implant loosening. The

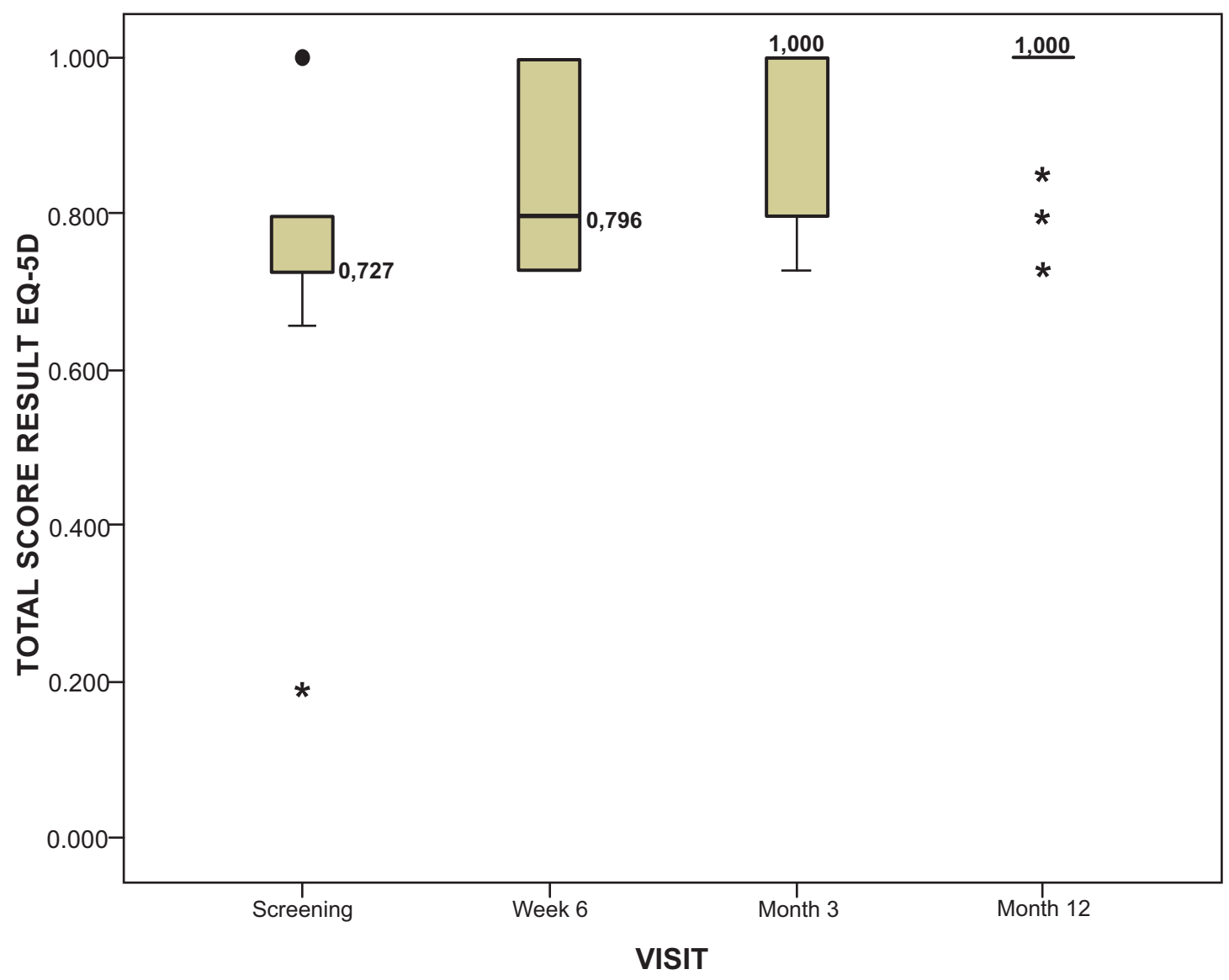

Fig. (10). EQ-5D results in the course of the study. The EQ-5D assessment 12 months postoperatively shows maximum score (1.0) in 24 patients, 0.73 for two patients, 0.80 for two patients and 0.85 for one patient. The improvements compared to the baseline values (screening) are significant (six weeks: $p=0.001$, three months: $p<0.001,12$ months: $p<0.001$ ), Wilcoxon Signed Ranks Test). 
SonicFusion technology was introduced by the manufacturer with the aim to enhance the anchoring capabilities. Until now, there is limited clinical experience with biodegradable polylactide implants fixated by ultrasonic melting. Eckelt $e t$ $a l$. reported on the successful use of resorbable pins inserted with the aid of ultrasound in cranioplasty for eight infants aged 12 months to four years with craniosynostosis [38]. They observed a considerably shorter application time compared to screw fixation with increased stability. Aldana et al. reported on the use of ultrasound-aided bone fixation with polylactide pins in 28 patients during craniotomies or craniofacial reconstructions [28]. They achieved adequate stability with few complications and concluded that the system was easy to use and provided rapid fixation of implants. Reichwein et al. reported on the application of the ultrasound-aided fixation of polylactide pins in 75 patients that were treated for fractures in the area of maxillofacial surgery [39]. They concluded that this fixation system shows general feasibility, sufficient mechanical stability, and efficient intraoperative handling for a wide variety of indications in craniomaxillofacial surgery.

In our study, the median surgery time was 33.5 minutes (range, 18 to 57 minutes) including concomitant forefoot procedures for hammer toe or bunionette deformity. Using screw osteosynthesis, skin-to-skin times between 42.5 minutes to 108 minutes have been reported in the past [40, 41]. Although a direct comparison is certainly not feasible, we feel able to state that there is so far no indication that the SonicPin technique prolongs theatre time.

The presented study was designed to assess the safety and performance of the use of SonicPins for the fixation of Austin/Chevron osteotomies. We did not find any major complications, the advancement of a MTP-1 joint arthritis most likely would have occurred with any other fixation method. In two cases $(6.7 \%)$ an incomplete melting of the pin, possibly due to improper function of the ultrasound hand piece, occurred. In both cases uneventful healing was observed. In one case (3.3\%), a slight lateral dislocation of the head fragment was noticed. The MRI revealed irregular imaging of the pin with signs of a step-off in the implant, which were interpreted as a fracture of the pin, although no discontinuity was evident (Fig. 4A, B). One of the cases presented showed a deformation of the pin in the MRI after three months. Postoperative X-rays showed an excessive lateral shift of the head fragment, which might have induced increased shear forces onto the pin (Fig. 4A, B). Due to the thickness of the slices given by the chosen MRI sequence a pin breakage cannot be fully excluded. While one of the cases showed signs of an intra-osseous edema around the pin after three months (Fig. 2A, B), the additional X-ray taken after three months showed intra-osseous sclerosis which led to the misinterpretation of the MRI (Fig. 3). The bony healing after 12 months was uneventful without any signs of foreign body reactions.

One of the aims of this study was to prove the absence of foreign body reactions. The chosen method of investigation was the MRI examination. The MRI was chosen to allow qualitative assessment of the polymer resorption behavior. MRI has been used successfully in the past to monitor bone healing and degradation of polylactide implants [42, 43]. As assessed by this method, no cases of detectable foreign body reactions occurred after 12 months. Also, no visible signs of resorption or degradation of the implants were seen, which leads to the conclusion that either the follow-up time has to be prolonged, or a better analytical method has to be determined.

The documented EQ-5D results demonstrate a significant improvement from a median value of 0.727 preoperatively to 1.0 after 12 months. Apart from one patient with increasing Hallux rigidus complaints, no additional complaints like pain or skin irritation were reported by the patients.

Strengths of our study are a homogenous study design and a clear focus on direct complications and results of the implants with a good radiological follow-up documentation as well as a focus on the changes in quality of life measure.

The limitations of our investigation are the missing functional scores, the limited sample size and a limited follow-up period. These items were not in the focus of the study design, nevertheless we think that future studies are required that should include a larger sample size and compare the SonicPin to standard fixation of an Austin/Chevron osteotomy with K-Wires or screws in a controlled manner to balance known and unknown factors that can influence the outcome. The follow-up period should possibly be extended to 24 months or 36 months to evaluate the further degradation behavior of the SonicPin system.

In conclusion, we could show that with an ultrasound activated biodegradable pin a Chevron osteotomy can be fixated in a safe and reliable manner assuring a satisfactory rise in the quality of life over the short term.

\section{CONFLICT OF INTEREST}

The authors confirm that this article content has no conflict of interest.

\section{ACKNOWLEDGEMENTS}

This study was partially funded by Stryker Osteosynthesis.

\section{REFERENCES}

[1] Austin DW, Leventen EO. A new osteotomy for hallux valgus: a horizontally directed "V" displacement ostoetomy of the metatarsal head for hallux valgus and primus cavus. Clin Orthop Relat Res 1981; 157: 25-30.

[2] Sorensen MD, Hyer CF. Metatarsus primus varus correction: the osteotomies. Clin Podiatr Med Surg 2009; 26(3): 409-25.

[3] Crosby LA, Bozarth GR. Fixation comparison for chevron osteotomies. Foot Ankle Int 1998; 19(9462912): 41-3.

[4] Hanft JR, Kashuk KB, Bonner AC, Toney M, Schabler J. Rigid internal fixation of the Austin/Chevron osteotomy with Herbert screw fixation: a retrospective study. J Foot Surg 1992; 31(5): 512-8.

[5] Barca F, Busa R. Austin/chevron osteotomy fixed with bioabsorbable poly-L-lactic acid single screw. J Foot Ankle Surg 1997; 36(1): 15-20.

[6] Leenslag JW, Pennings AJ, Bos RR, Rozema FR, Boering G. Resorbable materials of poly(L-lactide). VI. Plates and screws for internal fracture fixation. Biomaterials 1987; 8(1): 70-3.

[7] Schneider M, Eckelt U, Reitemeier B, et al. Stability of fixation of diacapitular fractures of the mandibular condylar process by ultrasound-aided resorbable pins (SonicWeld $\mathrm{Rx}(\mathrm{R})$ System) in pigs. Br J Oral Maxillofac Surg 2011; 49(4): 297-301. 
[8] Caminear DS, Pavlovich R Jr, Pietrzak WS. Fixation of the chevron osteotomy with an absorbable copolymer pin for treatment of hallux valgus deformity. J Foot Ankle Surg 2005; 44(3): 203-10.

[9] Claes LE, Ignatius AA, Rehm KE, Scholz C. New bioresorbable pin for the reduction of small bony fragments: design, mechanical properties and in vitro degradation. Biomaterials 1996; 17(16): $1621-6$.

[10] Pavlovich R Jr, Caminear D. Granuloma formation after chevron osteotomy fixation with absorbable copolymer pin: a case report. J Foot Ankle Surg 2003; 42(4): 226-9.

[11] Bos RR, Rozema FR, Boering G, Nijenhuis AJ, Verwey AB. Bioabsorbable plates and screws for internal fixation of mandibular fractures. A study in six dogs. Int J Oral Maxillofa Surg 1989; 18(6): 365-9.

[12] Esemenli T. Chevron osteotomy: a clinical and radiographic analysis. Foot Ankle Int 1998; 19(1): 60-1.

[13] Bos RR, Rozema FR, Boering G, Leenslag JW, Verwey AB, Pennings AJ. [Bioabsorbable poly(L-lactide) osteosynthesis plates and screws for the fixation of zygomatic bone fractures]. Dtsch $\mathrm{Z}$ Mund Kiefer Gesichtschir 1989; 13(6): 422-4.

[14] Viehe R, Haupt DJ, Heaslet MW, Walston S. Complications of screw-fixated chevron osteotomies for the correction of hallux abducto valgus. J Am Podiatr Med Assoc 2003; 93(6): 499-502.

[15] Olms K, Braemer A, Randt T, Radigk S, Schulz AP. Die Lapidus Arthrodese zur Korrektur des Hallux valgus. Fuss Sprunggelenk 2009; 7(3): 164-72.

[16] Stadlinger B, Pilling E, Mai R, et al. Effect of biological implant surface coatings on bone formation, applying collagen, proteoglycans, glycosaminoglycans and growth factors. J Mater Sci Mater Med 2008; 19(3): 1043-9.

[17] Rozema FR, Levendag PC, Bos RR, Boering G, Pennings AJ. Influence of resorbable poly(L-lactide) bone plates and screws on the dose distributions of radiotherapy beams. Int J Oral Maxillofac Surg 1990; 19(6): 374-6.

[18] Bos RR, Rozema FR, Boering G, Nijenhuis AJ, Pennings AJ, Jansen HW. Bone-plates and screws of bioabsorbable poly (Llactide) - an animal pilot study. Br J Oral Maxillofac Surg 1989; 27(6): 467-76.

[19] Bergsma JE, Rozema FR, Bos RR, Boering G, de Bruijn WC, Pennings AJ. In vivo degradation and biocompatibility study of in vitro pre-degraded as-polymerized polyactide particles. Biomaterials 1995; 16(4): 267-74.

[20] Ferguson SJ, Weber U, von Rechenberg B, Mayer J. Enhancing the mechanical integrity of the implant-bone interface with BoneWelding technology: determination of quasi-static interfacial strength and fatigue resistance. J Biomed Mater Res B Appl Biomater 2006; 77(16211571): 13-20.

[21] Langhoff JD, Kuemmerle JM, Mayer J, et al. An ultrasound assisted anchoring technique (bonewelding technology) for fixation of implants to bone - a histological pilot study in sheep. Open Orthop J 2009; 3(19572033): 40-7.

[22] Rozema FR, Bos RR, Boering G, Leenslag JW, Pennings AJ. Experimental fractures of the mandibular body of sheep and dogs. A new technique. Br J Oral Maxillofac Surg 1989; 27(2): 163-8.

[23] Pilling E, Mai R, Theissig F, Stadlinger B, Loukota R, Eckelt U. An experimental in vivo analysis of the resorption to ultrasound activated pins (Sonic weld) and standard biodegradable screws (ResorbX) in sheep. Br J Oral Maxillofac Surg 2007; 45(6): 447-50.

[24] Bergsma JE, de Bruijn WC, Rozema FR, Bos RR, Boering G. Late degradation tissue response to poly(L-lactide) bone plates and screws. Biomaterials 1995; 16(1): 25-31.

[25] Hanft JR, Kashuk KB, Bonner AC, Toney M, Schabler J. Rigid internal fixation of the Austin/Chevron osteotomy with Herbert screw fixation: a retrospective study. J Foot Surg 1992; 31(5): 512-8.

[26] Nkenke E, Vairaktaris E, Schwarz S, et al. Prospective assessment of complications associated with ultrasound activated resorbable pin osteosynthesis in pediatric craniofacial surgery: preliminary results. Neurocirugia 2011; 22(6): 498-506.

[27] Meara DJ, Knoll MR, Holmes JD, Clark DM. Fixation of Le Fort I Osteotomies With Poly-DL-Lactic Acid Mesh and Ultrasonic Welding-A New Technique. J Oral Maxillofac Surg 2012; 70(5): 1139-44.

[28] Aldana PR, Roy S, Postlethwait RA, James HE. Ultrasound-aided fixation of a biodegradable cranial fixation system: uses in pediatric neurosurgery. J Neurosurg Pediatr 2009; 3(5): 420-4.

[29] Nitsche M, Koy S, Mörz M, Koch R, Eckeu U. Exploration of tumor suppressors p16INK4a and p14ARF in oral leukoplakias. Mund Kiefer Gesichtschir 2007; 11(6): 317-26.

[30] Stadlinger B, Pilling E, Huhle $\mathrm{M}$, et al. Evaluation of osseointegration of dental implants coated with collagen, chondroitin sulphate and BMP-4: an animal study. Int J Oral Maxillofac Surg 2008; 37(1): 54-9.

[31] Arnoldi J, Henry P, Procter P, Robioneck B, Jönsson A. In vivo tissue response to ultrasound assisted application of biodegradable pins into cortical and cancellous bone structures: a histological and densitometric analysis in rabbits. J Biomater Sci Polym Ed 2012; 23(5): 663-76.

[32] Hurst NP. Re: Quality of life measures. Br J Rheumatol 1997; 36(1): $147-8$.

[33] Hurst NP, Kind P, Ruta D, Hunter M, Stubbings A. Measuring health-related quality of life in rheumatoid arthritis: validity, responsiveness and reliability of EuroQol (EQ-5D). Br J Rheumatol 1997; 36(5): 551-9.

[34] Coughlin, MJ, Thompson FM. The high price of high-fashion footwear. Instructional course lectures 1995; 44: 371-7.

[35] Sharma KM, Parks BG, Nguyen A, Schon LC. Plantar-to-dorsal compared to dorsal-to-plantar screw fixation for proximal chevron osteotomy: a biomechanical analysis. Foot Ankle Int 2005; 26(10): 854-8.

[36] Mai R, Lauer G, Pilling E, et al. Bone welding - a histological evaluation in the jaw. Ann Anat 2007; 189(4): 350-5.

[37] Porter MD, Anderson MG. Results of bioabsorbable fixation of metatarsal osteotomies. Am J Orthopedics 2004; 33(12): 609-11.

[38] Eckelt U, Nitsche M, Müller A, Pilling E, Pinzer T, Roesner D. Ultrasound aided pin fixation of biodegradable osteosynthetic materials in cranioplasty for infants with craniosynostosis. J Craniomaxillofac Surg 2007; 35(4-5): 218-21.

[39] Reichwein A, Schicho K, Moser D, et al. Clinical experiences with resorbable ultrasonic-guided, angle-stable osteosynthesis in the panfacial region. J Oral Maxillofac Surg 2009; 67(6): 1211-7.

[40] Crosby LA, Bozarth GR. Fixation comparison for chevron osteotomies. Foot Ankle International./American Orthopaedic Foot and Ankle Society [and] Swiss Foot and Ankle Society 1998; 19(1): 41-3.

[41] Armstrong DG, Pupp GR, Harkless LB. Our fixation with fixation: are screws clinically superior to external wires in distal first metatarsal osteotomies? J Foot Ankle Surg: Official Publication of the American College of Foot and Ankle Surgeons 1997; 36(5): 353-5; discussion 395-6.

[42] Kontio R, Ruuttila P, Lindroos L, et al. Biodegradable polydioxanone and poly $(1 / \mathrm{d})$ lactide implants: an experimental study on peri-implant tissue response. Int J Oral Maxillofac Surg 2005; 34(7): 766-76.

[43] Pihlajamaki H, Kinnunen J, Bostman O. In vivo monitoring of the degradation process of bioresorbable polymeric implants using magnetic resonance imaging. Biomaterials 1997; 18(19): 1311-5. 